\title{
Copper Pollution Increases the Resistance of Soil Archaeal Community to Changes in Water Regime
}

\author{
Jing $\mathrm{Li}^{1,2,3,4} \cdot$ Yu-Rong $\mathrm{Liu}^{3} \cdot \mathrm{Li}-J u a n \mathrm{Cui}^{1,4}$ • Hang-Wei Hu${ }^{5}$ • Jun-Tao Wang ${ }^{3}$. \\ Ji-Zheng $\mathrm{He}^{3,5}$
}

Received: 6 March 2017 / Accepted: 27 April 2017 /Published online: 10 May 2017

(C) Springer Science+Business Media New York 2017

\begin{abstract}
Increasing efforts have been devoted to exploring the impact of environmental stresses on soil bacterial communities, but the work on the archaeal community is seldom. Here, we constructed microcosm experiments to investigate the responses of archaeal communities to the subsequent dryrewetting (DW) disturbance in two contrasting soils (fluvoaquic and red soil) after 6 years of copper pollution. Ten DW cycles were exerted on the two soils with different copper levels, followed by a 6-week recovery period. In both soils, archaeal diversity (Shannon index) in the high copper-level treatments increased over the incubation period, and archaeal community structure changed remarkably as revealed by the non-metric multidimensional scaling ordinations. In both soils, copper pollution altered the response of dominant operational taxonomic units (OTUs) to the DW disturbance. Throughout the incubation and recovery period, the resistance
\end{abstract}

Electronic supplementary material The online version of this article (doi:10.1007/s00248-017-0992-0) contains supplementary material, which is available to authorized users.

Yu-Rong Liu

yrliu@rcees.ac.cn

1 Institute of Wetland Research, Chinese Academy of Forestry, Beijing 100091, China

2 Beijing Key Laboratory of Wetland Ecological Function and Restoration, Beijing, China

3 State Key Laboratory of Urban and Regional Ecology, Research Center for Eco-Environmental Sciences, Chinese Academy of Sciences, Beijing 100085, China

4 Beijing Hanshiqiao National Wetland Ecosystem Research Station, Beijing 101399, China

5 Faculty of Veterinary and Agricultural Sciences, The University of Melbourne, Parkville, VIC 3010, Australia of archaeal abundance to the DW disturbance was higher in the copper-polluted soils than soils without pollution. Taken together, copper pollution altered the response of soil archaeal diversity and community composition to the DW disturbance and increased the resistance of the archaeal abundance. These findings have important implications for understanding soil microbial responses to ongoing environmental change.

Keywords Archaea $\cdot$ Abundance $\cdot$ Resistance $\cdot$ Community composition $\cdot$ Copper pollution $\cdot$ Dry-rewetting disturbance

\section{Introduction}

Soil drying and rewetting (DW) is a common type of environmental disturbance in agricultural practices, exemplified by the frequencies and intensities of irrigation. The significant fluctuations in soil conditions induced by the DW alternations might notably affect the soil-dwelling microorganisms [1-3], which contribute to the sustainability of ecosystem functioning and services. When confronted with drought, some microorganisms can survive from the water-limited conditions by accumulating osmoregulatory solutes [1]. Once the dry soil being rewetted, sensitive microorganisms would be dying by bursting of cells due to the sudden increase in water potential, while microbes with high tolerance might quickly release the accumulated solutes to survive $[2,3]$. Therefore, it is necessary to elucidate the responses of soil microbial communities to the DW alternations, which can improve our ability to predict the responses of ecosystem functions to future environmental disturbances.

There has been some evidence suggesting that the DW disturbance indeed notably affects different soil microorganisms. For instance, the diffusion of substrates in dry soils can be hindered by water limitation, which most probably inhibited soil nitrification activity carried out by the nitrifying 
microorganisms [3]. However, the rewetting event could result in an instantaneous flush of nutrients (e.g., carbon and nitrogen) into soil solution, stimulating the increase of soil respiration [4]. The DW cycles significantly reduced the production and emission of methane in a paddy soil, compared with continuous flooding, by decreasing the methanogen $m c r A$ gene transcripts and by changing composition of active methanogens [5]. These studies suggested that the DW disturbance is an important factor driving the changes in soil microbial community structure and activities. However, it is still lack of studies on how soil microbial community exposed to environmental stress (e.g., heavy metal pollution) will respond to the DW disturbance.

It is in debate whether the microbial community under a primary stress consistently responds to a subsequent disturbance [6]. For example, soil microorganisms in heavy metal-contaminated soils were less resistant to a subsequent heat or salt stress compared with those in the uncontaminated soils, which was explained by the less energy of the stressed microorganisms to cope with the additional stress [7]. However, ammonia oxidizers in a long-term zinc-contaminated soil were found to be more resistant to a secondary zinc stress than those in the uncontaminated soil, because the zinc-exposed microorganisms might have developed zinc tolerance in the long-term zinc pollution [8]. The effects of a primary stress on soil microbial community to a subsequent stress may be determined by the similarity of the primary and subsequent disturbances [9]. Previous studies demonstrated that long-term copper pollution in the field increased the resistance of soil microorganisms to a subsequent copper stress, whereas it reduced the resistance of soil bacteria to a subsequent DW disturbance in soil microcosm experiments $[4,10]$. Most of studies above mainly focused on the changes of bacteria other than archaea in response to primary and subsequent environmental disturbances, due to the numerous dominances of bacteria in the soil microbial community $[4,11]$. The responses of archaeal community to disturbances remain largely unknown.

Increasing evidence, however, suggested that archaea play irreplaceable roles in sustaining the ecosystem function [12]. For example, the ammonia-oxidizing archaea predominate among ammonia-oxidizing prokaryotes in soils $[13,14]$ and play a more important role than ammoniaoxidizing bacteria in nitrification of acidic soils [15-17]. In addition, as an important group of archaea, methanogenic archaea are involved in the methanogenesis process which contributes to approximately 1 billion tons of methane per year produced from the anoxic environments [18]. Despite the functional importance of archaea, however, our knowledge is lacking regarding the responses of soil archaeal communities to the DW alternations.

The main objective of this study was to explore the response of the archaeal community in copper-contaminated soils to subsequent DW disturbances. Approximately 3.4 million tons of copper are released to the terrestrial surface every year [19] and most of the copper is produced by anthropogenic activities. Few studies have been carried out to explore how the soil archaeal communities in coppercontaminated soils respond to subsequent DW disturbance. Soils collected from two long-term experimental field sites were incubated in a microcosm experiment exerted by ten cycles of DW alternations. Soil archeal abundance, diversity, and community composition were analyzed through quantitative PCR and high-throughput sequencing. We hypothesized that copper pollution could affect the resistance of soil archaea to the subsequent DW disturbance.

\section{Materials and Methods}

\section{Site Description and Soil Sampling}

Soil samples were collected from two field experimental sites situated in Dezhou (DZ, 37.33 $\left.{ }^{\circ} \mathrm{N}, 116.63^{\circ} \mathrm{E}\right)$ and Qiyang (QY, $\left.26.75^{\circ} \mathrm{N}, 111.88^{\circ} \mathrm{E}\right)$. The soil in Dezhou is classified as a fluvo-aquic soil which was alkaline $(\mathrm{pH}=7.9)$ with a ratio of clay/silt/sand (18:18:64) and in Qiyang belong to acidic red soil $(\mathrm{pH}=4.3)$ with a ratio of clay/silt/sand (46:35:19). In July 2007, three treatments with different copper levels were established in randomized plots with four replicates. Owing to the different soil properties (in particular, soil $\mathrm{pH}$ ) in the two experimental sites, it is assumed that a large proportion of $\mathrm{Cu}^{2+}$ precipitates as $\mathrm{Cu}_{2}(\mathrm{OH})_{2} \mathrm{CO}_{3}$ and $\mathrm{Cu}(\mathrm{OH})_{2}$ [20] in the alkaline soil. Therefore, a gradient of higher copper concentrations $(0$, $800,3200 \mathrm{mg} \mathrm{Cu} \mathrm{kg}^{-1}$ soil) in the fluvo-aquic soil was designed to create comparable toxicity effects compared with a gradient of copper concentrations $(0,200,800 \mathrm{mg}$ $\mathrm{Cu} \mathrm{kg}^{-1}$ soil) in the red soil. Copper chloride $\left(\mathrm{CuCl}_{2}\right)$ powder was mixed with the top 20-cm surface soils and applied only once in 2007. In subsequent years, the maize/wheat rotation was planted in the plots with conventional field management practices. In July 2012, soil samples were taken from the top $10 \mathrm{~cm}$ by mixing five soil cores $(10 \mathrm{~cm} \times 10 \mathrm{~cm})$ in each plot, transported on ice to the laboratory, and stored at $4{ }^{\circ} \mathrm{C}$ before the construction of soil microcosms. The physical-chemical characteristics and copper concentrations in both soils were listed in Table S1, and the photos of two sites were showed in Fig. S1.

\section{Soil Microcosm Incubation}

In this study, DW alternations were applied in soils with three different copper levels, and six treatments were established in microcosms including $\mathrm{Cu} 0-\mathrm{S}$ (S represent 
the DW stress), $\mathrm{Cu} 800-\mathrm{S}$, and $\mathrm{Cu} 3200-\mathrm{S}$ in the fluvo-aquic soil and $\mathrm{Cu} 0-\mathrm{S}, \mathrm{Cu} 200-\mathrm{S}$, and $\mathrm{Cu} 800-\mathrm{S}$ in the red soil. Soils were adjusted to $40 \%$ water-holding capacity (WHC) and pre-incubated in loosely capped vials at $25^{\circ} \mathrm{C}$ for 15 days. Thereafter, 108 microcosms $(6$ treatments $\times 6$ time points $\times 3$ replicates) were established in $100-\mathrm{ml}$ vials containing $10 \mathrm{~g}$ of soils and loosely capped to allow air exchange. The soils of three replicates were from the replicate plots in the field. The DW disturbance included ten DW cycles $(1 \mathrm{c}-10 \mathrm{c})$ with drying for $24 \mathrm{~h}$ (fan-forced air at $25^{\circ} \mathrm{C}$ ) and rewetting (adjusted to $60 \%$ WHC by adding sterilized water) for $48 \mathrm{~h}$ [21]. The soil moisture was maintained by constant weighing. Soil water content was determined immediately after drying or rewetting by oven drying the soil subsamples at $105{ }^{\circ} \mathrm{C}$ for $8 \mathrm{~h}$, and it was below $5 \%$ after drying and recovered to $30 \%$ after rewetting. In the period of the DW disturbance, microcosms were incubated at $25{ }^{\circ} \mathrm{C}$ in the dark for 30 days. In order to explore the recovery of the soil archaeal community, the microcosms were incubated at $25{ }^{\circ} \mathrm{C}$ in the dark for another 42 days after the DW disturbance. Soils were destructively sampled after $0,1 \mathrm{c}, 6 \mathrm{c}$, and $10 \mathrm{c}$ of the DW disturbance and 1 week $(1 \mathrm{w})$ and 6 weeks (6w) during the recovery period. The whole soil core was collected from the containers and then mixed homogeneously, and there was no $\mathrm{Cu}$ leaching in our experiments. The soil samples were freeze-dried and stored at $-80{ }^{\circ} \mathrm{C}$ before DNA extraction.

\section{Soil DNA Extraction and Quantitative PCR Analysis}

The total DNA was extracted from $0.25 \mathrm{~g}$ of soil using the MoBio PowerSoil DNA Isolation Kit (MoBio Laboratories, Carlsbad, CA, USA) following the manufacturer's instructions. The cell-lysis step was performed on a FastPrep bead beating system (Bio-101, Vista, CA, USA) at a speed of $5.5 \mathrm{~m} \mathrm{~s}^{-1}$ for $30 \mathrm{~s}$. The quality and quantity of the isolated DNA were assessed using a NanoDrop® ND-2000c UV-Vis spectrophotometer (NanoDrop Technologies, Wilmington, DE, USA).

The archaeal 16S rRNA gene abundance was quantified on an iCycler iQ 5 thermocycler (BioRad Laboratories, Hercules, CA, USA) using the primer pair A364aF and A934bR [11]. The $25-\mu$ l reaction mixture contained $12.5 \mu$ of SYBR Premix Ex Taq (Takara Biotechnology, Dalian, China), $0.5 \mu \mathrm{l}$ of each primer $(10 \mu \mathrm{M})$, and $2 \mu \mathrm{l}$ of fivefold diluted DNA template (1-10 ng). Amplification conditions were as follows: $94{ }^{\circ} \mathrm{C}$ for $1 \mathrm{~min}, 40$ cycles of $20 \mathrm{~s}$ at $94{ }^{\circ} \mathrm{C}, 30 \mathrm{~s}$ at $59{ }^{\circ} \mathrm{C}$, and $30 \mathrm{~s}$ at $72{ }^{\circ} \mathrm{C}$, followed by plate reads at $83{ }^{\circ} \mathrm{C}$. Standard curves were generated using tenfold serial dilutions of plasmid containing correct insert of the archaeal 16S rRNA gene, and the PCR efficiency for different assays ranged between 90 and $105 \%$.
High-Throughput Profiling of the Archaeal Community by Illumina Sequencing

The primers of 519f and 915r were used for the amplification of the archaeal 16S rRNA gene [22]. The 20- $\mu$ l PCR reaction mixture consisted of $0.4 \mu \mathrm{l}$ of FastPfu Polymerase (TransGen Biotech, China), $4 \mu$ l of $5 \times$ FastPfu Buffer, $2 \mu$ of dNTPs $(2.5 \mu \mathrm{M}), 0.6 \mu \mathrm{l}$ of each primer $(5 \mu \mathrm{M})$, fivefold diluted template DNA $(\sim 15 \mathrm{ng})$, and sterilized water. Thermal cycling conditions were as follows: an initial denaturation of $5 \mathrm{~min}$ at $95{ }^{\circ} \mathrm{C}, 30$ cycles of $30 \mathrm{~s}$ at $95{ }^{\circ} \mathrm{C}, 30 \mathrm{~s}$ at $55^{\circ} \mathrm{C}$, and $45 \mathrm{~s}$ at $72{ }^{\circ} \mathrm{C}$, with a final elongation of $72{ }^{\circ} \mathrm{C}$ for $10 \mathrm{~min}$. The PCR products were purified with a Wizard SV Gel and PCR CleanUp System (Promega, San Luis Obispo, CA, USA), and the concentrations were measured using the Qubit dsDNA HS Assay Kit (Invitrogen, Carlsbad, CA, USA). The purified PCR products were combined in approximately equimolar amount into a single tube and sent for sequencing on the Illumina Miseq platform (Illumina, San Diego, CA, USA).

The obtained raw sequences were assigned to individual samples, quality filtered, and de-noised using Quantitative Insights Into Microbial Ecology (QIIME) 1.7.0 following the standard operation procedure [23]. UPARSE was used to identify and remove chimeric sequences [24] and to pick operational taxonomic units (OTUs) at the $97 \%$ sequence similarity from the quality reads on the Bio-Linux platform [25]. A set of representative sequences from each OTU was aligned against the Greengenes database [26] using PyNAST [27], and the taxonomic classification was conducted using the Ribosome Database Project Classifier [28]. Resampling according to the minimum sequence numbers per sample (1001) across all samples was performed before the downstream analysis (Fig. S2).

\section{Statistical Analysis}

The archaeal diversity was characterized by calculating the Shannon index in QIIME 1.7.0. Shifts in the archaeal community composition were visualized by the non-metric multidimensional scaling (NMDS) ordinations based on the BrayCurtis dissimilarity matrix. The heat maps displaying the variations of the relative abundance of dominant OTUs were generated using the "gplots" package in the R platform. The significance of the impact of the DW disturbance on the archaeal community compositions was tested by permutational multivariate analysis of variance (PerMANOVA) using the "vegan" package with 999 permutations in the R platform. The redundancy analysis (RDA) was used to explain the effects of soil physical-chemical characteristics on the archaeal community. One-way analysis of variance was performed to compare the relative abundances of archaeal taxa, Shannon index, and the archaeal 16S rRNA gene abundance across different treatments in SPSS 19 (IBM, Armonk, NY, USA). Statistically significant differences were accepted at $P<0.05$. 
We calculated the resistance index (RS) of the archaeal and bacterial abundance using the following equation [29]:

$\operatorname{RS}\left(t_{0}\right)=1-\frac{2\left|D_{0}\right|}{\left(C_{0}+\left|D_{0}\right|\right)}$

where $D_{0}$ is the difference between the control $\left(C_{0}\right.$; values of the abundance at day 0 ) and the disturbed soils (values of the abundance at $1 \mathrm{c}, 6 \mathrm{c}, 10 \mathrm{c}, 1 \mathrm{w}$, and $6 \mathrm{w})$. This index has the advantage of being standardized by the control and being bounded between -1 (less resistance) and +1 (maximal resistance), and it remains bounded even when extreme values are encountered [29]. The calculation of RS index of the bacterial abundance was based on the data of bacterial abundance in our previous study [4] which is showed in the supplementary materials (Fig. S3). The RS index of the archaeal abundance was calculated by using the data collected in this study.

\section{Results}

\section{Impacts of the DW Disturbance on the Archaeal Diversity and Community Compositions}

Illumina Miseq sequencing was employed to investigate the impacts of the DW disturbance on the archaeal community compositions. After resampling across all samples, 1001 of sequences were obtained for analyzing the archaeal communities. The diversity (Shannon index) of archaeal community fluctuated slightly from 2.39 to 3.28 in the fluvo-aquic soil compared with the changes from 0.29 to 3.42 in the red soil (Fig. 1a, b). In the fluvo-aquic soil, Shannon index in the Cu3200-S treatment had lower variation compared with the other two treatments during the incubation (Fig. 1a).
However, in the red soil, Shannon index in all three treatments changed significantly during the incubation, with increasing tendency observed in the Cu800-S treatment and decreasing tendencies observed in the $\mathrm{Cu} 0-\mathrm{S}$ and $\mathrm{Cu} 200-\mathrm{S}$ treatments. NMDS ordinations based on the Bray-Curtis dissimilarity matrices revealed notable shifts of archaeal community structure during the incubation period especially in the $\mathrm{Cu} 800-\mathrm{S}$ treatment in the red soil and Cu3200-S treatment in the fluvo-aquic soil (Fig. 2a, b). Results were further corroborated by the PerMANOVA analysis $(P<0.05)$. The archaeal communities were dominated by the Nitrososphaera, which belonged to Nitrososphaerales class within the Thaumarchaeota phylum in the fluvo-aquic soil, in which the percentage exceeded $90 \%$ (Fig. 3a). The dominant taxa of Nitrososphaera increased after $1 \mathrm{c}$ but decreased after $1 \mathrm{w}$ in both $\mathrm{Cu} 0-\mathrm{S}$ and $\mathrm{Cu} 800-\mathrm{S}$ treatments. However, the relative abundance of Nitrososphaera changed slightly in the Cu3200-S treatment. Similarly, except in the Cu800-S treatment, Nitrososphaera remained the most abundant taxa in the red soil, followed by Methanocella which belonged to Methanomicrobia class within the Euryarchaeota phylum (Fig. 3b). The relative abundances of Nitrososphaera and Methanocella remained largely unchanged during the incubation in the $\mathrm{Cu} 0-\mathrm{S}$ and $\mathrm{Cu} 200-\mathrm{S}$ treatments. The archaeal community composition in the $\mathrm{Cu} 800-\mathrm{S}$ treatment significantly changed and became more diverse with dominant taxa numbers increased. This result was also consistent with the significant increase of diversity in the Cu800-S treatment. The relative abundance of Methanocella significantly increased after the DW disturbance while Nitrososphaera and Methanomassiliicoccus decreased in response to the DW disturbance. The redundancy analysis (RDA) revealed that the differences in the archaeal community between two soils were determined by $\mathrm{pH}$, SOM, and TN (Fig. S4, $P<0.05$ ). The first and second axes could explain $63 \%$ of the variance.

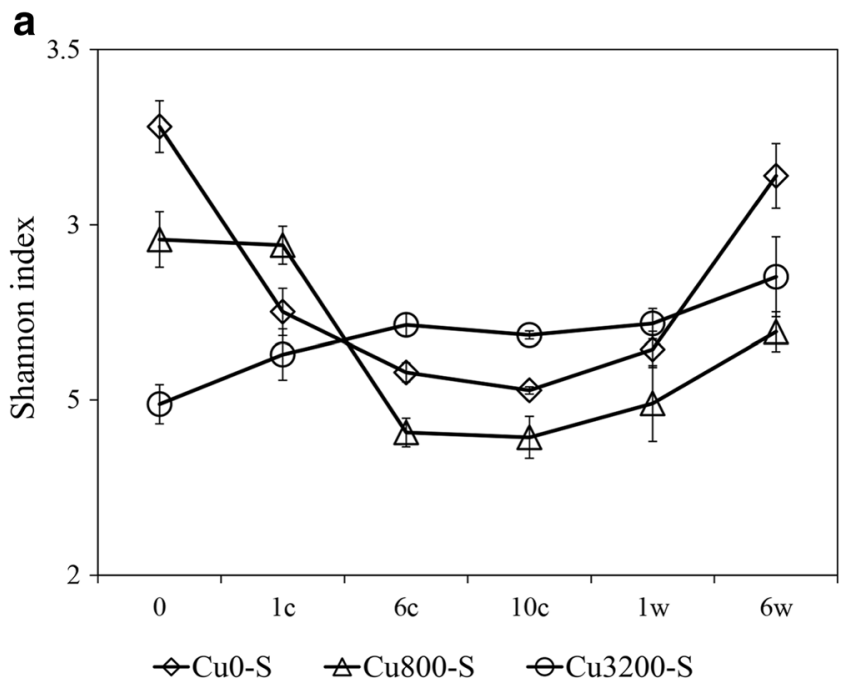

b

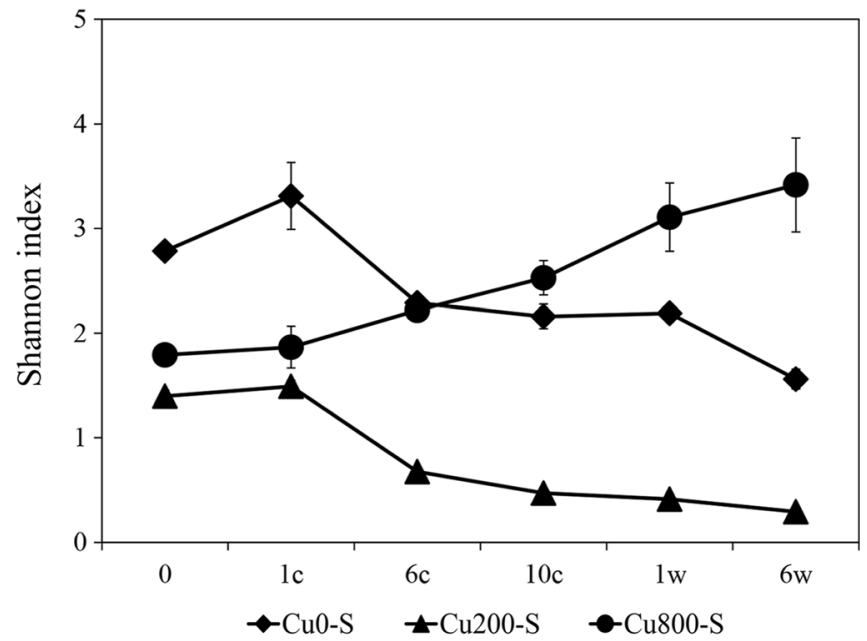

Fig. 1 Changes of Shannon index among different treatments in the fluvo-aquic soil (a) and the red soil (b) with the repeated cycles of DW alternations $(1 \mathrm{c}, 6 \mathrm{c}$, and $10 \mathrm{c})$ and in the recovery period $(1 \mathrm{w}$ and $6 \mathrm{w})$. Error bars represent standard errors $(n=3)$ 
Fig. 2 NMDS ordinations derived from the Bray-Curtis dissimilarity matrices display the shifts in the archaeal community among different treatments in the fluvo-aquic soil (a) and the red soil (b) during the incubation period
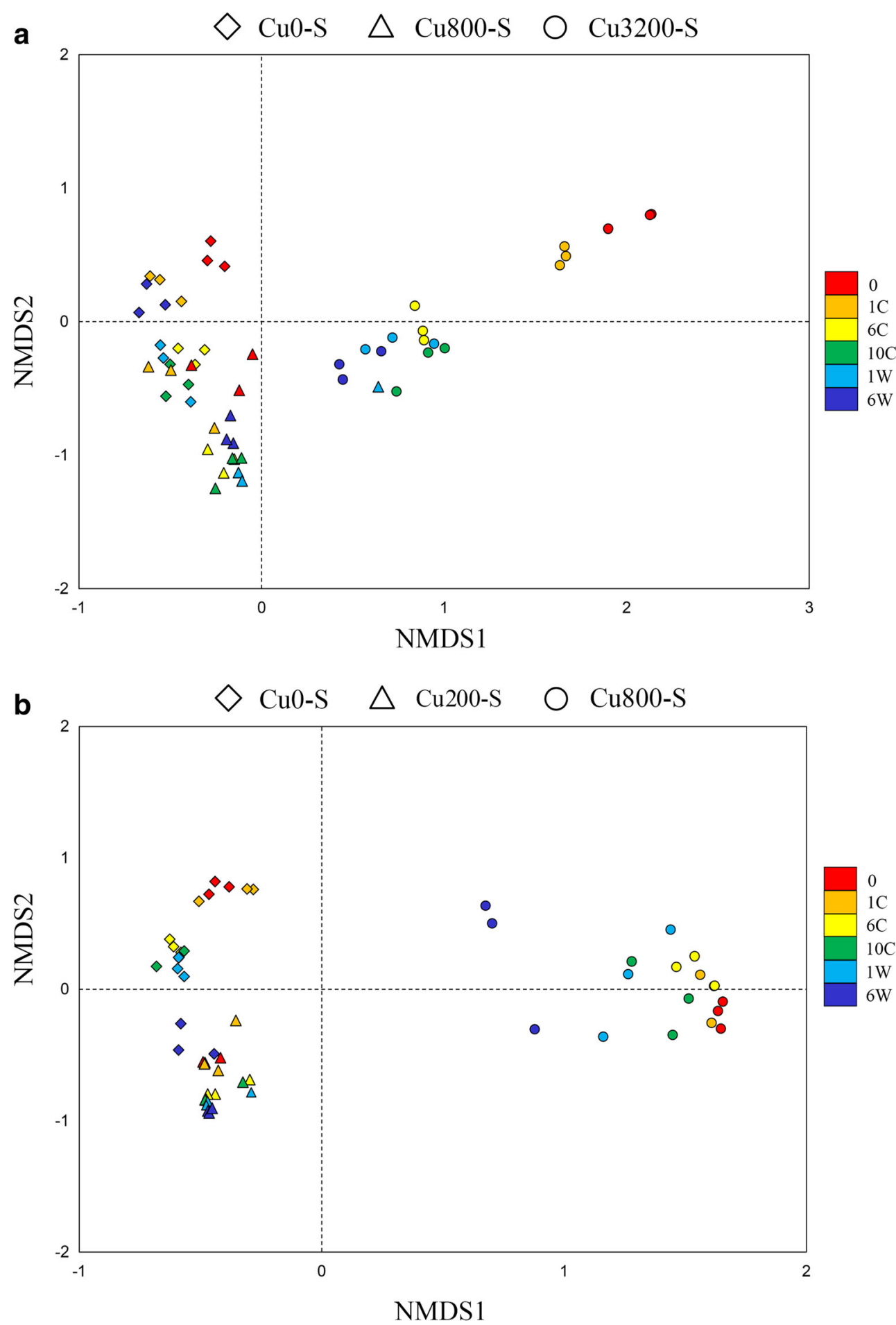

The heatmaps illustrate that the changes of the relative abundances of dominant OTUs in both soils (Fig. 4a, b), and OTUs with the relative abundances above $10 \%$ were identified as dominant OTUs. In the fluvo-aquic soil, although the archaeal community composition on the genus level did not significant change during the incubation period, the variations of dominant OTUs were significant especially in the Cu3200-S treatment (Fig. 4a). The relative abundance of OTU-10 was higher in the $\mathrm{Cu} 3200-\mathrm{S}$ treatment than that in other two treatments. Furthermore, the relative abundance of dominant OTUs belonging to Nitrososphaera viennensis such as OTU-11, OTU8 , and OTU-4 increased after $6 \mathrm{c}$, while OTU-10 classified as Nitrososphaera gargensis decreased significantly with increasing DW alternations (1c, 6c, 10c) and in the recovery period (1w, 6w) (Fig. 4a and Table S2). The dominant OTUs also fluctuated significantly in the red soil (Fig. 4b). The relative 
Fig. 3 Shifts in the archaeal community composition on the genus level among different treatments in the fluvo-aquic soil (a) and the red soil (b) during the incubation period. Error bars represent standard errors $(n=3)$
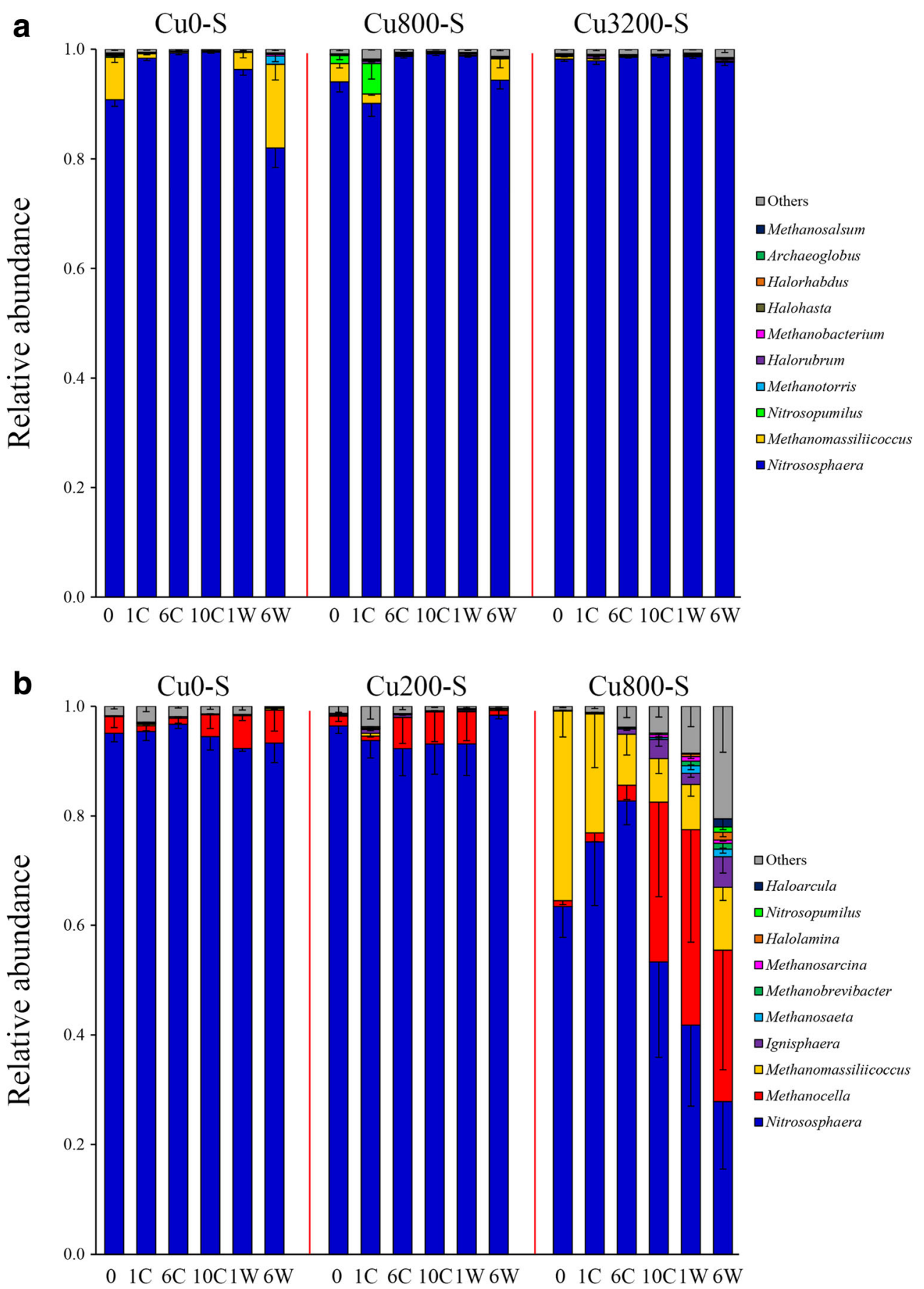

abundance of OTU-1 (N. viennensis) significantly increased after $6 \mathrm{c}$ including $1 \mathrm{w}$ and $6 \mathrm{w}$ in $\mathrm{Cu} 0-\mathrm{S}$ and $\mathrm{Cu} 200-\mathrm{S}$ treatments. The numbers and relative abundance of dominant OTUs in the Cu800-S treatment also differed from those in the $\mathrm{Cu} 0-\mathrm{S}$ and $\mathrm{Cu} 200-\mathrm{S}$ treatments which were consistent with the results on the genus level. The relative abundance of OTU-5 (N. viennensis), OTU-26 (Methanocella arvoryzae), OTU2133 ( N. viennensis), and OTU-14 (Methanomassiliicoccus luminyensis) in the Cu800-S treatment significantly increased resulting in the increase of dominant OTU numbers (Fig. 4b and Table S2). Moreover, the relative abundance of these dominant OTUs changed significantly during the incubation period with the significant increase of OTU-26 and decrease of OTU5, OTU-2133, and OTU-14 after 1w.

\section{Impacts of the DW Disturbance on the Archaeal} Abundance and the Resistance of the Abundance

The archaeal abundance ranging from $1.66 \times 10^{8}$ to $2.07 \times 10^{9}$ copies $\mathrm{g}^{-1}$ soil was significantly higher in the 
Fig. 4 Variations of the relative abundance of dominant OTUs in the archaeal community in the fluvo-aquic soil (a) and the red soil (b) during the incubation period

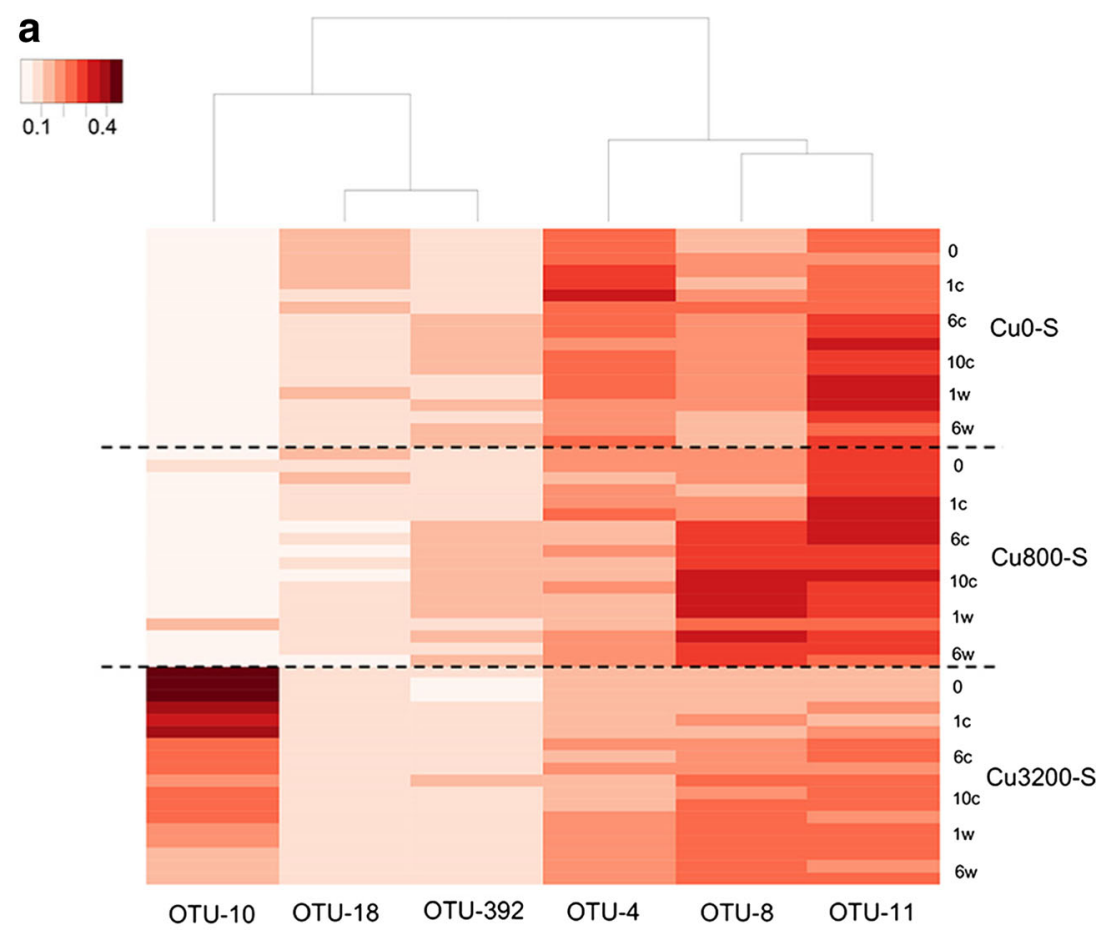

b

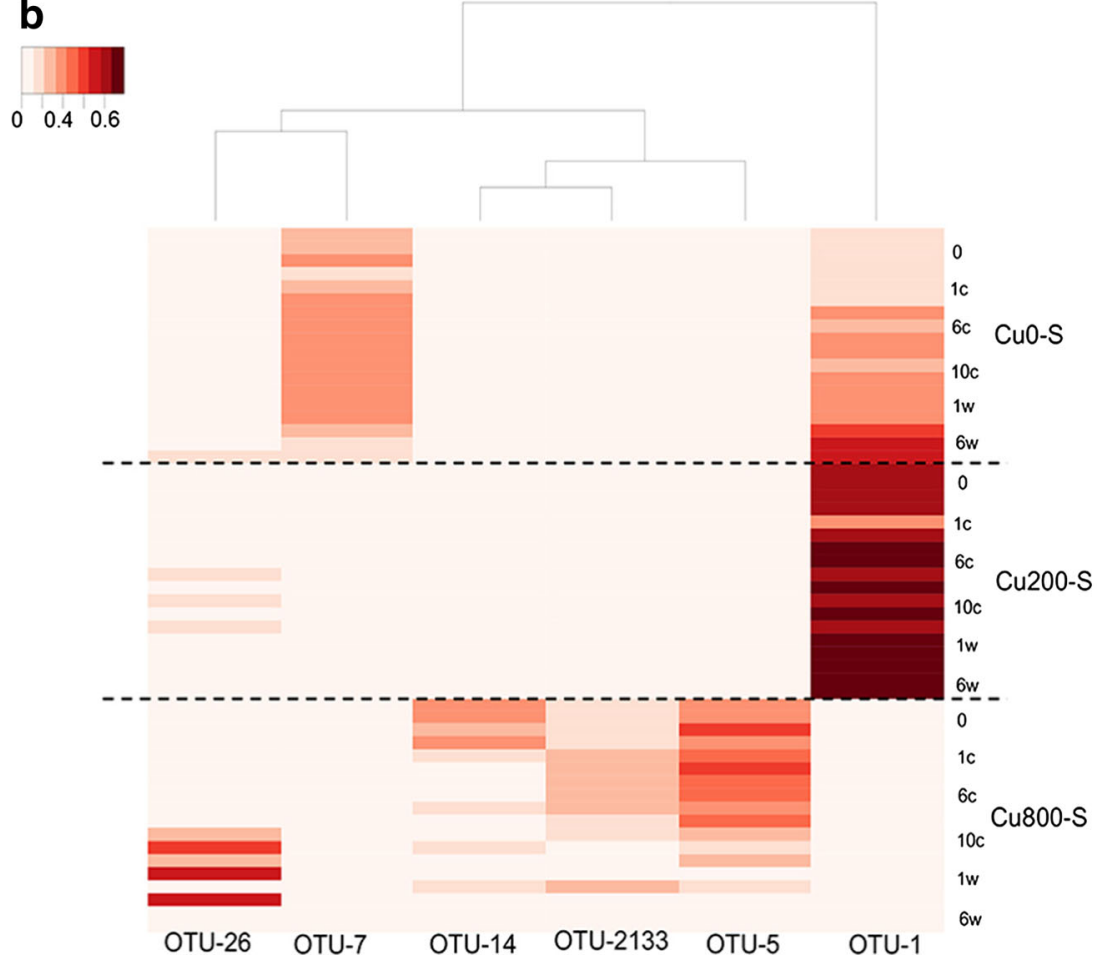

fluvo-aquic soil (Fig. 5a) than that (from $5.14 \times 10^{6}$ to $3.71 \times 10^{8}$ copies $^{-1}$ soil) in the red soil (Fig. 5c). Except the $\mathrm{Cu} 0-\mathrm{S}$ treatments in both soils, the archaeal abundance of all $\mathrm{Cu}$-polluted treatments (including Cu200-S, Cu800-S in the red soil, and $\mathrm{Cu} 800-\mathrm{S}, \mathrm{Cu} 3200-\mathrm{S}$ in the fluvo-aquic soil) fluctuated slightly during the incubation period (Fig. 5a, c). As for the Cu0-S treatments in both soils, the archaeal abundance was significantly higher than those in other treatments and significantly increased after six cycles of DW disturbance (6c) (Fig. 5a, c).

The values of the RS index for archaeal abundance in both soils were higher in the $\mathrm{Cu}$-polluted treatments (including $\mathrm{Cu} 200-\mathrm{S}$ and $\mathrm{Cu} 800-\mathrm{S}$ in the red soil and $\mathrm{Cu} 800-\mathrm{S}$ and $\mathrm{Cu} 3200-\mathrm{S}$ in the fluvo-aquic soil) than those in the $\mathrm{Cu} 0-\mathrm{S}$ treatments especially in the recovery period (1w and $6 \mathrm{w}$ ) (Fig. 5b, d). The RS index of Cu0-S treatments in both soils 
decreased after $6 \mathrm{c}$. As for the values of the RS index for bacterial abundance, the RS index in both soils began to be higher in the Cu-polluted treatments (including Cu200-S and $\mathrm{Cu} 800-\mathrm{S}$ in the red soil and $\mathrm{Cu} 800-\mathrm{S}$ and $\mathrm{Cu} 3200-\mathrm{S}$ in the fluvo-aquic soil) than those in the $\mathrm{Cu} 0-\mathrm{S}$ treatments after $1 \mathrm{w}$ (Fig. 6a, b). The ranges of the RS index for archaeal and bacterial abundance were from -0.6 to 0.9 in both soils, which was within the scope of -1 to 1 according to the equation.

\section{Discussion}

Increasing evidence suggested that higher diversity can promote the community-level stability [30, 31]. In our study, the archaeal diversity in the highest-Cu pollution red soil (Cu800$\mathrm{S})$ significantly increased in the recovery period after repeated

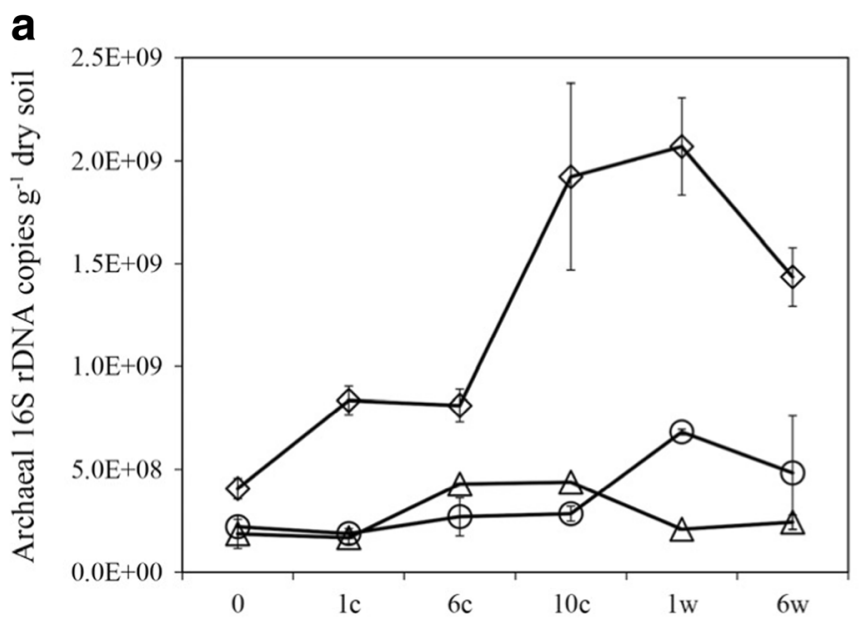

b

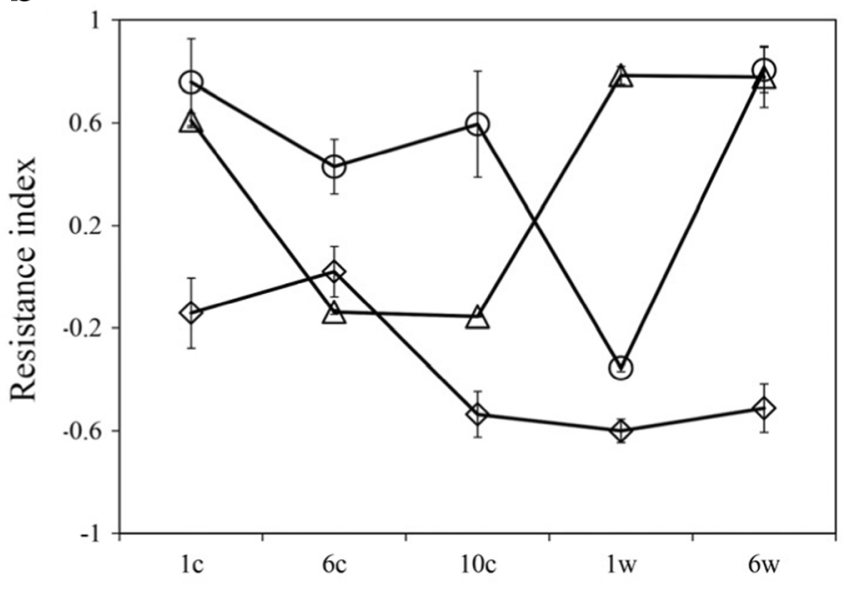

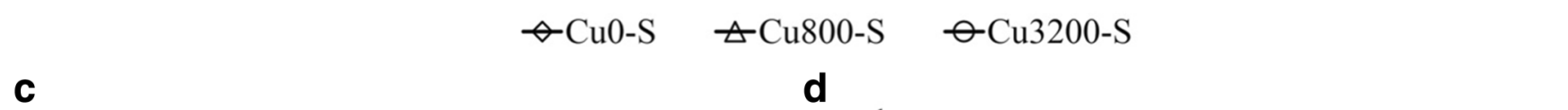

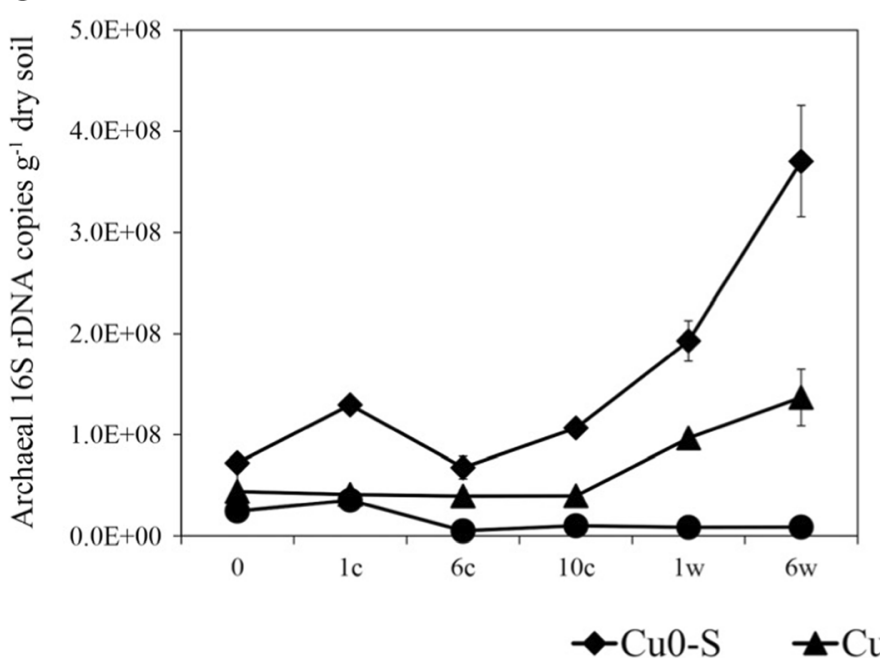

Fig. 5 Changes of the archaeal abundance among different treatments in the fluvo-aquic soil (a) and the red soil (c) with the repeated cycles of DW alternations (1c, 6c, and 10c) and in the recovery period (1w and $6 \mathrm{w})$. The

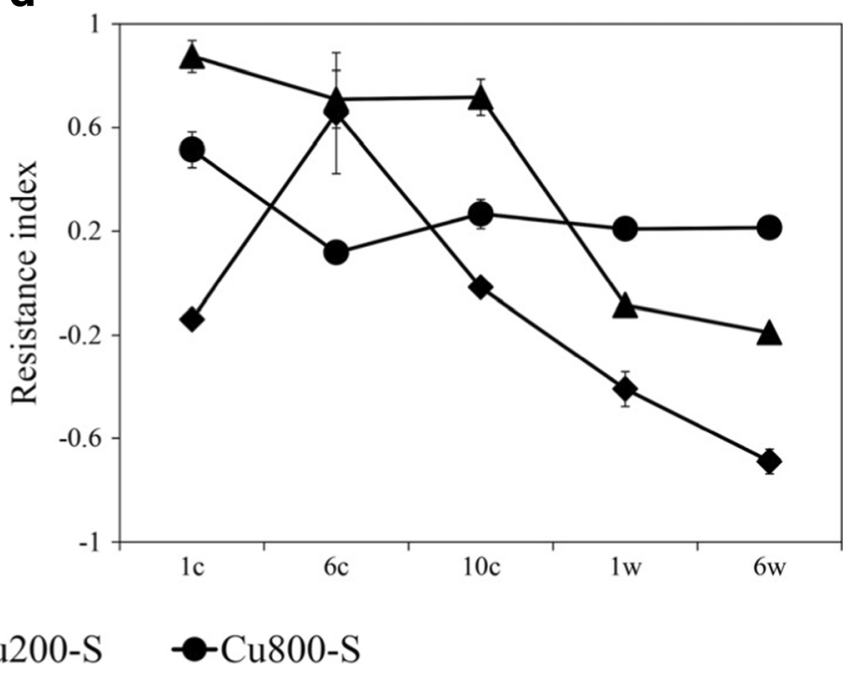

shifts of the RS index of the archaeal abundance among different treatments in the fluvo-aquic soil (b) and the red soil (d). Error bars represent standard errors $(n=3)$ 

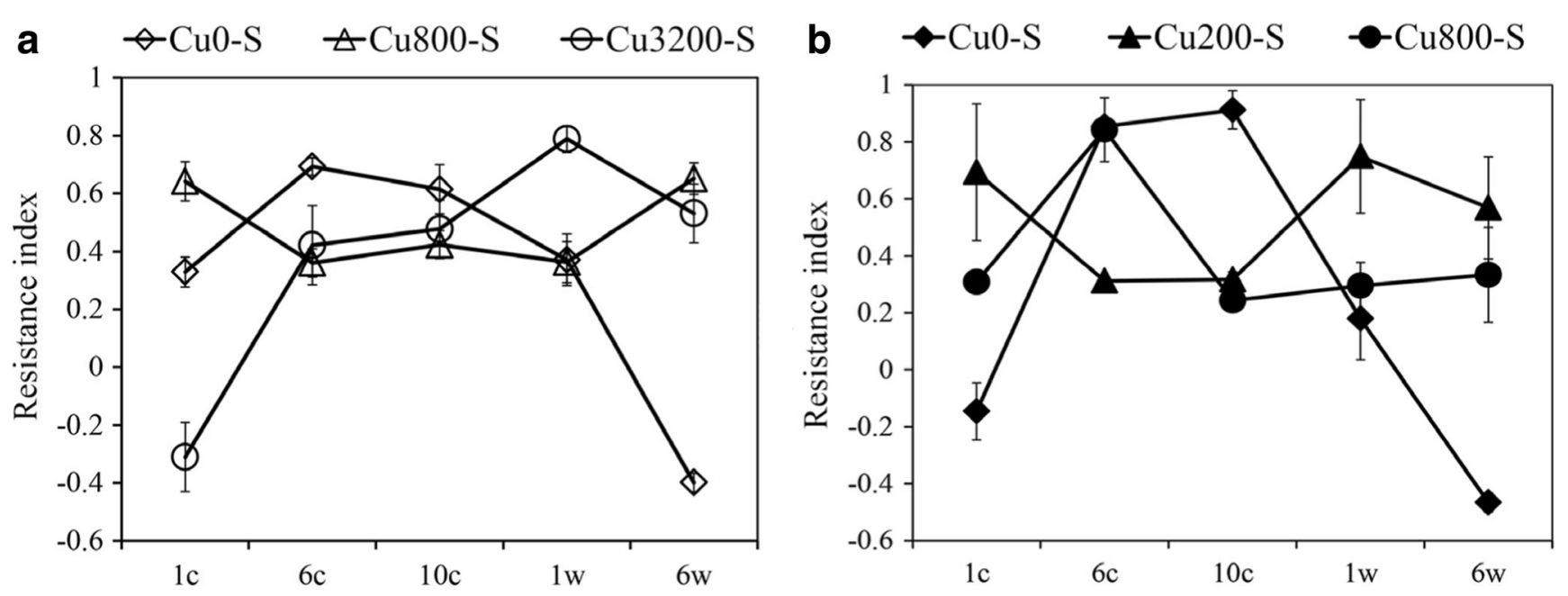

Fig. 6 The shifts of the RS index of the bacterial abundance among different treatments in the fluvo-aquic soil (a) and the red soil (b). Error bars represent standard errors $(n=3)$

possesses a Cu-translocating P-type ATPase (CopA homologue), which might be involved in $\mathrm{Cu}$ tolerance [33]. In addition, all Thaumarchaeota including Nitrososphaera might contain another putative $\mathrm{Cu}$ transport system (CopC-CopD) which provides additional resistance to $\mathrm{Cu}$ [34]. Therefore, Nitrososphaera was also the dominant archaeal taxa in the Cu-polluted soils. Despite the dominance of Nitrososphaera in both soils, the Methanocella was another dominant group in the red soil. Methanocella appear to be widely distributed in aerated upland soils but colonizes rice roots in particular [35]. However, once the soils are flooded, the core taxa of the Methanocella become rapidly active. The differences of the $\mathrm{pH}, \mathrm{SOM}$, and TN in two soil resulted in the different archaeal community compositions (Fig. S4).

The heavy metal pollution could remarkably alter the response of microbial community structure to subsequent disturbance $[4,36]$ and was supported by our results of significant changes of the dominant OTUs in the archaeal community after the DW disturbance in the Cu-polluted soils (Fig. 4a, b). Some indicator species were observed induced by $\mathrm{Cu}$ pollution and the DW disturbance. In the high Cu-polluted fluvoaquic soil, OTU-11, OTU-8, and OTU-4 classified as $N$. viennensis might be tolerant to the DW disturbance in the recovery period while OTU-10 classified as $N$. gargensis showed sensitivity. As for the high $\mathrm{Cu}$-polluted red soil, OTU-26 belonging to Methanocella was tolerant while OTU-5, OTU-2133, and OTU-14 were sensitive to the DW disturbance. The numbers of dominant OTUs increased by copper pollution, which lead to the high diversity after DW disturbance in $\mathrm{Cu}$-polluted soils. Above all, our results revealed that copper pollution might increase the number of nitrifiers and methanogens in response to the DW disturbance in the upland soils.

Long-term heavy metal pollution could induce the tolerance of soil microorganisms [37, 38], which could also increase the resistance of microorganisms to the subsequent disturbance. Our results support the notion that although the exposure of $\mathrm{Cu}$ pollution decreased the archaeal abundance, it increased the resistance of the archaeal abundance to the subsequent DW disturbance especially in the recovery period in two contrasting soils. The archaeal abundance in both soils exposed to $\mathrm{Cu}$ pollution displayed small fluctuations to the DW disturbance, which agreed with the finding that copper pollution had little impact on the archaeal abundance in the marine sediments [39]. Previous study revealed that $\mathrm{Cu}$ pollution decreases the resistance of substrate-induced respiration to the DW disturbance [4]. However, the resistance of substrate-induced respiration to the DW disturbance was based on the variations during the period of the repeated DW cycles without consideration of the recovery period after the DW disturbance. In this study, Cu pollution decreased the resistance of bacterial and archaeal abundance to the DW disturbance in the period of repeated DW alterations in both soils, while increased the resistance of them in the recovery period. The bacterial or archaeal abundance potentially indicates the biomass of bacteria or archaea, therefore, the microbial abundance might be closely related with the activity of microorganisms [40]. It suggested that the tolerance of archaeal community induced by $\mathrm{Cu}$ pollution revealed by the increase of archaeal diversity and tolerant species might increase its resistance to the DW disturbance [39].

Archaeal abundance in the $\mathrm{Cu}$-polluted soil changed slightly after DW disturbance in this study compared with the bacterial abundance exposed to the $\mathrm{Cu}$ pollution [4], which was supported by lower resistance of bacterial abundance in the $\mathrm{Cu}$-polluted soil than in the non-polluted soil during the DW disturbance period (Fig. 6). It might be supported by the differences of extracellular osmolality tolerance between bacteria and Archaea, which result in higher sensitivity of bacteria to the moisture shift than archaea [41]. Therefore, archaea could 
tolerate higher extracellular osmolality compared with bacteria [42], and archaea may have stronger resistance to dryrewetting disturbance. These results implied that the effect of primary stress on the responses of microorganisms to the subsequent disturbance was still unclear. The resistance of microbial community might be determined by the types and frequencies of disturbances and was also dependent on different microorganisms.

\section{Conclusions}

In conclusion, $\mathrm{Cu}$ pollution significantly affected the responses of archaeal diversity and community composition to the DW disturbance and increased the resistance of archaeal abundance after the DW disturbance. Specifically, the response of dominant OTUs to subsequent DW disturbance in $\mathrm{Cu}$-polluted soils was different from those in the non-polluted soils. The resistance of the archaeal abundance was higher in the $\mathrm{Cu}$-polluted soils especially in the recovery period after DW disturbance than that in soils without pollution, owing to the increase of archaeal diversity and tolerant species induced by $\mathrm{Cu}$ pollution. These findings advance our understanding of soil microbial responses to environmental stress. Owing to limitations of microcosm experiment, however, future field study should strengthen our prediction of the impact of environmental disturbance on soil microbiome.

Acknowledgements This work was financially supported by the Fundamental Research Funds for the Central Non-profit Research Institution of CAF (CAFYBB2016QA020)and the Strategic Priority Research Program Chinese Academy of Sciences (XDB15020200).

\section{References}

1. Yao SH, Zhang B, Hu F (2011) Soil biophysical controls over rice straw decomposition and sequestration in soil: the effects of drying intensity and frequency of drying and wetting cycles. Soil Biol. Biochem. 43:590-599

2. Hu HW, Chen D, He JZ (2015) Microbial regulation of terrestrial nitrous oxide formation: understanding the biological pathways for prediction of emission rates. FEMS Microbiol. Rev. 39:729-749

3. Hu HW, Macdonald CA, Trivedi P, Holmes B, Bodrossy L, He JZ, Singh BK (2015) Water addition regulates the metabolic activity of ammonia oxidizers responding to environmental perturbations in dry subhumid ecosystems. Environ. Microbiol. 17:444-461

4. Li J, Wang JT, Hu HW, Ma YB, Zhang LM, He JZ (2016) Copper pollution decreases the resistance of soil microbial community to subsequent dry-rewetting disturbance. J. Environ. Sci. 39:155-164

5. Ma K, Conrad R, Lu Y (2012) Responses of methanogen mcrA genes and their transcripts to an alternate dry/wet cycle of paddy field soil. Appl. Environ. Microbiol. 78:445-454

6. Philippot L, Cregut M, Chèneby D, Bressan M, Dequiet S, MartinLaurent F, Ranjard L, Lemanceau P (2008) Effect of primary mild stresses on resilience and resistance of the nitrate reducer community to a subsequent severe stress. FEMS Microbiol. Lett. 285:51-57

7. Tobor-Kapłon MA, Bloem J, Römkens PF, de Ruiter PC (2006) Functional stability of microbial communities in contaminated soils near a zinc smelter (Budel, The Netherlands). Ecotoxicology 15: $187-197$

8. Mertens J, Springael D, deTroyer I, Cheyns K, Wattiau P, Smolders E (2006) Long-term exposure to elevated zinc concentrations induced structural changes and zinc tolerance of the nitrifying community in soil. Environ. Microbiol. 8:2170-2178

9. Griffiths BS, Philippot L (2012) Insights into the resistance and resilience of the soil microbial community. FEMS Microbiol. Rev. 37:112-129

10. Li J, Zheng YM, Liu YR, Ma YB, Hu HW, He JZ (2014) Initial copper stress strengthens the resistance of soil microorganisms to a subsequent copper stress. Microb. Ecol. 67:931-941

11. Fierer N, Schimel JP (2002) Effects of drying-rewetting frequency on soil carbon and nitrogen transformations. Soil Biol. Biochem. 34:777-787

12. Cao P, Zhang LM, Shen JP, Zheng YM, Di HJ, He JZ (2012) Distribution and diversity of archaeal communities in selected Chinese soils. FEMS Microbiol. Ecol. 80:146-158

13. He JZ, Shen JP, Zhang LM, Zhu YG, Zheng YM, Xu MG, Di HJ (2007) Quantitative analyses of the abundance and composition of ammonia-oxidizing bacteria and ammonia-oxidizing archaea of a Chinese upland red soil under long-term fertilization practices. Environ. Microbiol. 9:2364-2374

14. Leininger S, Urich T, Schloter M, Schwark L, Qi J, Nicol G, Prosser J, Schuster S, Schleper C (2006) Archaea predominate among ammonia-oxidizing prokaryotes in soils. Nature 442:806-809

15. He JZ, Hu HW, Zhang LM (2012) Current insights into the autotrophic thaumarchaeal ammonia oxidation in acidic soils. Soil Biol. Biochem. 55:146-154

16. $\mathrm{Hu} \mathrm{HW}, \mathrm{Xu} \mathrm{ZH}, \mathrm{He}$ JZ (2014) Ammonia-oxidizing archaea play a predominant role in acid soil nitrification. Adv. Agron. 125:261302

17. Zhang LM, Hu HW, Shen JP, He JZ (2012) Ammonia-oxidizing archaea have more important role than ammonia-oxidizing bacteria in ammonia oxidation of strongly acidic soils. ISME J 6:1032-1045

18. Thauer RK, Kaster AK, Seedorf H, Buckel W, Hedderich R (2008) Methanogenic archaea: ecologically relevant differences in energy conservation. Nat Rev Microbiol 6:579-591

19. Zhou ZY, Fan YP, Wang MJ (2000) Heavy metal contamination in vegetables and their control in China. Food Rev Int 16:239-255

20. Ma Y, Lombi E, Oliver IW, Nolan AL, McLaughlin MJ (2006) Long-term aging of copper added to soils. Environ Sci Technol 40:6310-6317

21. Degens BP, Schipper LA, Sparling GP, Duncan LC (2001) Is the microbial community in a soil with reduced catabolic diversity less resistant to stress or disturbance? Soil Biol. Biochem. 33:11431153

22. Herfort L, Schouten S, Abbas B, Veldhuis MJ, Coolen MJ, Wuchter C, Boon JP, Herndl GJ, Damsté JSS (2007) Variations in spatial and temporal distribution of archaea in the North Sea in relation to environmental variables. FEMS Microbiol. Ecol. 62:242-257

23. Caporaso JG, Kuczynski J, Stombaugh J, Bittinger K, Bushman FD, Costello EK, Fierer N, Pena AG, Goodrich JK, Gordon JI (2010) QIIME allows analysis of high-throughput community sequencing data. Nat. Methods 7:335-336

24. Edgar RC (2013) UPARSE: highly accurate OTU sequences from microbial amplicon reads. Nat. Methods 10:996-998

25. Field D, Tiwari B, Booth T, Houten S, Swan D, Bertrand N, Thurston M (2006) Open software for biologists: from famine to feast. Nat. Biotechnol. 24:801-804

26. DeSantis TZ, Hugenholtz P, Larsen N, Rojas M, Brodie EL, Keller K, Huber T, Dalevi D, Hu P, Andersen GL (2006) Greengenes, a 
chimera-checked 16S rRNA gene database and workbench compatible with ARB. Appl. Environ. Microbiol. 72:5069-5072

27. Caporaso JG, Bittinger K, Bushman FD, DeSantis TZ, Andersen GL, Knight R (2010) PyNAST: a flexible tool for aligning sequences to a template alignment. Bioinformatics 26:266-267

28. Wang Q, Garrity GM, Tiedje JM, Cole JR (2007) Naive Bayesian classifier for rapid assignment of rRNA sequences into the new bacterial taxonomy. Appl. Environ. Microbiol. 73:5261-5267

29. Orwin KH, Wardle DA (2004) New indices for quantifying the resistance and resilience of soil biota to exogenous disturbances. Soil Biol. Biochem. 36:1907-1912

30. Hooper DU, Chapin III FS, Ewel JJ, Hector A, Inchausti P, Lavorel S, Lawton JH, Lodge DM, Loreau M, Naeem S, Schmid B, Setälä H, Symstad AJ, Vandermeer J, Wardle DA (2005) Effects of biodiversity on ecosystem functioning: a consensus of current knowledge. Ecol. Monogr. 75:3-35

31. Yang H, Jiang L, Li LH, Li A, Wu MY, Wan SQ (2012) Diversitydependent stability under mowing and nutrient addition: evidence from a 7-year grassland experiment. Ecol. Lett. 15:619-626

32. Nielsen UN, Ayres E, Wall DH, Bardgett RD (2011) Soil biodiversity and carbon cycling: a review and synthesis of studies examining diversity-function relationships. Eur. J. Soil Sci. 62:105-116

33. Chan H, Babayan V, Blyumin E, Gandhi C, Hak K, Harake D, Kumar K, Lee P, Li TT, Liu HY (2010) The p-type ATPase superfamily. J. Mol. Microbiol. Biotechnol. 19:5-104

34. Spang A, Poehlein A, Offre P, Zumbrägel S, Haider S, Rychlik N, Nowka B, Schmeisser C, Lebedeva EV, Rattei T (2012) The genome of the ammonia-oxidizing Candidatus Nitrososphaera gargensis: insights into metabolic versatility and environmental adaptations. Environ. Microbiol. 14:3122-3145

35. Angel R, Claus P, Conrad R (2012) Methanogenic archaea are globally ubiquitous in aerated soils and become active under wet anoxic conditions. The ISME J 6:847-862

36. Griffiths BS, Philippot L (2013) Insights into the resistance and resilience of the soil microbial community. FEMS Microbiol. Rev. 37:112-129

37. Rusk JA, Hamon RE, Stevens DP, McLaughlin MJ (2004) Adaptation of soil biological nitrification to heavy metals. Environ Sci Technol 38:3092-3097

38. Ruyters S, Mertens J, Springael D, Smolders E (2010) Stimulated activity of the soil nitrifying community accelerates community adaptation to Zn stress. Soil Biol. Biochem. 42:766-772

39. Besaury L, Ghiglione JF, Quillet L (2014) Abundance, activity, and diversity of archaeal and bacterial communities in both uncontaminated and highly copper-contaminated marine sediments. Mar. Biotechnol. 16:230-242

40. Bai R, Xi D, He JZ, Hu HW, Fang YT, Zhang LM (2015) Activity, abundance and community structure of anammox bacteria along depth profile $\mathrm{s}$ in three different paddy soils. Soil Biol. Biochem. 91:212-221

41. Vasileiadis S, Coppolecchia D, Puglisi E, Balloi A, Mapelli F, Hamon RE, Daffonchio D, Trevisan M (2012) Response of ammonia oxidizing bacteria and archaea to acute zinc stress and different moisture regimes in soil. Microb. Ecol. 64:1028-1037

42. Martin DD, Ciulla RA, Roberts MF (1999) Osmoadaptation in archaea. Appl. Environ. Microbiol. 65:1815-1825 